\title{
Lower airway microbiota in children with Down syndrome compared to controls with similar respiratory symptomatology
}

\author{
Mariska De Lausnay ${ }^{1}$, Stijn Verhulst ${ }^{1,2}$, Lieve Boel $^{1}$, Kim Van Hoorenbeeck $^{1,2}$ \\ ${ }^{1}$ Department of Pediatrics, Antwerp University Hospital, Edegem, Belgium; ${ }^{2}$ Lab of Experimental Medicine and Pediatrics, Faculty of Medicine and \\ Health Sciences, University of Antwerp, Antwerpen, Belgium \\ Contributions: (I) Conception and design: K Van Hoorenbeeck, M De Lausnay; (II) Administrative support: L Boel; (III) Provision of study \\ materials or patients: S Verhulst, L Boel, K Van Hoorenbeeck; (IV) Collection and assembly of data: M De Lausnay, L Boel; (V) Data analysis and \\ interpretation: M De Lausnay; (VI) Manuscript writing: All authors; (VII) Final approval of manuscript: All authors. \\ Correspondence to: Mariska De Lausnay, MD. Antwerp University Hospital, Drie Eikenstraat 655, 2650 Edegem, Belgium. \\ Email: Mariska.DeLausnay@uza.be.
}

\begin{abstract}
Background: Children with Down syndrome (DS) often present with chronic or recurrent respiratory symptoms and generally have a more severe and prolonged disease course in case of infection. This can be caused by anatomical and/or immunological predisposition. With this study, we aim to compare microbial composition in the lower airways of patients with DS versus controls, to see if we can explain the difference in disease course.
\end{abstract}

Methods: All endoscopic procedures under general anesthesia in patients with DS were reviewed retrospectively. We compared the microbiological data from bronchoalveolar lavage fluid (BALF) cultures (when available) to a cohort of children with chronic respiratory symptoms but without any other relevant medical history.

Results: Endoscopic data were available for 65 DS patients, BAL cultures for 47 out of 65 patients (72\%). The "control" group consisted of 150 children without significant underlying disease, who were matched for age and sex. BAL culture results were available for 135 out of 150 patients (90\%). Microorganisms were categorized and compared between both groups, with no statistical differences. Among the microorganisms tested, the most frequently reported were typical bacteria such as Haemophilus influenzae, Moraxella catarrhalis, Streptococci and Staphylococci.

Conclusions: No significant differences in lower airways microbial composition of children with DS and chronic respiratory symptoms were found when compared to controls presenting similar symptomatology. A suggestion for future research may be to investigate possible differences in drug sensitivity.

Keywords: Down syndrome (DS); bronchoscopy; microbiota; lower airway symptoms

Submitted Dec 17, 2020. Accepted for publication May 25, 2021.

doi: $10.21037 /$ tp-20-460

View this article at: https://dx.doi.org/10.21037/tp-20-460

\section{Introduction}

Trisomy 21 or Down syndrome (DS) is a relatively common human genetic disorder (1). It is well known for the characteristic dysmorphic features, cognitive impairment and hypotonia, but patients also have a high incidence of congenital anomalies in several organ systems: most frequently affected are the heart, gastrointestinal tract and respiratory system (1). These features make them more prone to airway obstruction and other respiratory problems (2-5). Children with DS are more vulnerable to infections possibly

^ ORCID: Mariska De Lausnay, 0000-0003-2393-7317; Stijn Verhulst, 0000-0002-1922-9716. 
due to, among other factors, an altered immune status $(3,4)$. Studies show that respiratory problems such as upper and lower respiratory tract infections (e.g., otitis media, tonsillitis, pneumonia, bronchiolitis) are the most common admission diagnoses in this patient population $(6,7)$.

In a previous study (8), we focused on the anatomy of the lower airways. We reviewed endoscopic results to compare the prevalence of airway anomalies in a population of children with DS with a control group (both with chronic or recurrent respiratory symptoms). In conclusion, we found a significantly higher prevalence of both isolated and combined airway malformations compared to controls (with the occurrence of one or more anomalies in $71 \%$ of endoscopies in DS versus $32 \%$ of controls, $\mathrm{P}<0.001$ ). This confirmed the conclusions of previous small-scale studies (9-11). However, little is known about the microbiota of these children as we found no previous study concerning the microbial composition of the lower respiratory tract in DS patients.

In the past, the lungs were thought to be a sterile environment. Nowadays, it is widely accepted that the lower airways harbor a complex and diverse microbiota that differs substantially from the upper airways, which in turn represents different sub-niches such as the nasal or oral cavity (12). However, examining the lower airway microbiota is challenging (13). This is because of the technical difficulties in accurate sampling and also the extremely low bacterial burden in healthy lungs. While the upper respiratory tract has a high bacterial burden due to continuous exposure via ingestion and inhalation, this is 100 to 10,000 times lower in the lower airways. Although the vocal cords act as a barrier, there are still continuous bacterial challenges due to microaspiration, postnasal drip, regurgitation, hematogenous spread and inhalation (12). In addition to these exposures, use of antibiotics or steroids, coinfection with viruses and availability of nutrients can play important roles in the shaping of a specific microbiome and immunological phenotypes (12).

Studies in children with chronic cough and diagnosis of protracted bacterial bronchitis have shown clinically significant levels of the following microorganisms in bronchoalveolar lavage (BAL) fluid: Haemophilus influenzae, Streptococcus pneumoniae, Moraxella catarrbalis and Staphylococcus aureus (13-15). These pathogens were associated with altered bacterial community structure (lower alpha diversity of the respiratory microbiota), higher bacterial biomass and higher inflammatory parameters such as neutrophil percentage and several interleukins, compared to controls $(13,14)$.

A few case reports from the 1980-1990's describe atypically severe lower airway infections in children with DS. Cant et al. (16) described a series of four DS patients presenting with acute bacterial tracheitis. Three of them had cultures positive for Haemophilus influenzae and one remained sterile, probably due to sampling done after several doses of antibiotic therapy. All of them were severely ill and required mechanical ventilation. Orlicek et al. (17) described another series of three young DS patients with severe bilateral pneumonia with Mycoplasma pneumonia as causative agent. A report by Winters et al. (18) described a case of lethal pneumonia in a DS patient caused by Bordetella bronchiseptica, which is a microorganism generally found in animal species but in rare cases also causes severe infections in immunocompromised patients. These reports suggest a susceptibility in DS patients for atypical microorganisms or atypical course of infection. However, these reports are rare and non-recent. Further data are lacking.

Therefore, the aim of this study is to evaluate if the difference in prevalence, severity and duration of respiratory tract infections can be (partially) explained by comparing BAL fluid cultures between a cohort of children with DS from our institution (Antwerp University Hospital, Belgium), to a group of children with respiratory symptoms but without any other significant medical history. This may provide valuable information for future decision-making in terms of treatment (e.g., for choosing empirical antibiotic therapy). We present the following article in accordance with the Materials Design Analysis Reporting (MDAR) checklist (available at https://dx.doi.org/10.21037/tp-20-460).

\section{Methods}

Information gathered in databases from our previous study was considered. Retrospective chart review of all endoscopic procedures (flexible bronchoscopy and flexible/rigid laryngoscopy, all under general anesthesia with spontaneous breathing) was performed in the Antwerp University Hospital, Belgium, in pediatric patients with DS from April 2011 until June 2019 (8). As a control group, children without significant underlying disorders undergoing endoscopy for similar indications were selected from charts dating from January 2012 until January 2015. These databases are only accessible to the investigators and were approved by the Ethics Committee of our institution (Antwerp University Hospital, Belgium; approval number 19/17/229). Given the data are collected retrospectively and anonymized, informed 
Table 1 Patient characteristics of the study populations

\begin{tabular}{|c|c|c|c|c|}
\hline & Total $(n=182)$ & DS $(n=47)$ & Controls $(n=135)$ & Comparison ( $\mathrm{P}$ value) \\
\hline Sex (\% boys) & $116(63.7 \%)$ & $31(66 \%)$ & $85(63 \%)$ & 0.713 \\
\hline Airway anomaly present & $72(39.6 \%)$ & $32(68.1 \%)$ & $40(29.6 \%)$ & $<0.001$ \\
\hline Symptomatology (reason for bronchoscopy) & & & & 0.005 \\
\hline Recurrent infections & $41(22.5 \%)$ & $19(40.4 \%)$ & $22(16.3 \%)$ & \\
\hline Persistent radiographic abnormalities & $31(17 \%)$ & $7(14.9 \%)$ & $24(17.8 \%)$ & \\
\hline Respiratory failure & $17(9.3 \%)$ & $5(10.6 \%)$ & $12(8.9 \%)$ & \\
\hline Stridor, recurrent laryngitis & $16(8.8 \%)$ & $2(4.3 \%)$ & $14(10.4 \%)$ & \\
\hline
\end{tabular}

$P$ values calculated by Mann-Whitney $U$ test (age), Chi square test (sex, presence of airway anomaly) or Fisher exact test (symptomatology); significance level $\mathrm{P} \leq 0.05$.

consent was omitted. The study was conducted in accordance with the Declaration of Helsinki (as revised in 2013).

For each subject, availability of BAL sample obtained during the endoscopic procedure for its further microbiological investigation was checked in their medical records. If this was the case, results of cultures and/or PCR testing were listed as reported in the patient file. Immediately after sampling, the BAL samples are processed as follows. After a fraction is taken aside for PCR testing or viral culture (when requested), the remaining material is quantitatively inoculated on several culture media (all media are from bioMérieux Marcy-l'Étoile, France): a fixed volume of the sample $(0.1 \mathrm{~mL})$ is being inoculated on (I) a blood agar (BA) for detection of most gram-negative and gram-positive bacteria (except for some fastidious organisms), (II) a colistin nalidixic acid agar (CNA) for detection of gram-positive bacteria, (III) a MacConkey agar for detection of gram-negative bacilli and (IV) a HAEM agar for Haemophilus influenza and other fastidious organisms. The remainder of the sample is being centrifuged and used for microscopic evaluation and more specific cultures (e.g., for fungi and mycobacteria). BAs, CNA agars and HAEM agars are incubated at $37{ }^{\circ} \mathrm{C}$ in $5 \% \mathrm{CO}_{2}$ and MacConkey agars in ambient air at $35-37^{\circ} \mathrm{C}$. Cultures are assessed by well-trained lab technicians after 24 and 48 hours of incubation and relevant organisms identified by MALDI-TOF mass spectrometry. In case of doubt, further identification will be performed using 16s rRNA sequencing.

\section{Statistical analysis}

All positive cultures (even with small or indeterminable numbers of colony forming units per $\mathrm{mL}$ ) were taken into account, in order to determine both colonization and infection. These results were subsequently categorized and compared by statistical analysis in SPSS. We used a MannWhitney $\mathrm{U}$ test to compare ages between the two cohorts and Chi square test (or Fisher exact test when appropriate) to compare the two cohorts in terms of sex distribution, reason for endoscopic evaluation and microbial composition. The level of statistical significance was set at $\mathrm{P} \leq 0.05$.

\section{Results}

At present, over 300 children with DS are followed-up in our center by a multidisciplinary team. For 65 of them data on airway endoscopy are available. Our control group consists of 150 children with respiratory symptoms that warranted endoscopic evaluation, but without additional underlying conditions. BAL samples for cultures and PCR testing were obtained in 47 of the DS patients $(47 / 65=72 \%)$ and in 135 of our control patients $(135 / 150=90 \%)$. The groups are matched in terms of age and sex, with an overall mean age at time of bronchoscopy of 3.4 years and the majority being male (63.7\%). Reasons for endoscopic evaluation for both groups include recurrent infections, chronic cough or noisy breathing, persisting radiographic 


\begin{tabular}{|c|c|c|}
\hline & $\begin{array}{l}\text { Down } \\
(\mathrm{n}=47)\end{array}$ & $\begin{array}{c}\text { Controls } \\
(n=135)\end{array}$ \\
\hline \multicolumn{3}{|l|}{ (A) Typical bacteria } \\
\hline Haemophilus influenzae & 29 & 55 \\
\hline Moraxella catarrhalis & 13 & 29 \\
\hline Staphylococcus aureus & 5 & 13 \\
\hline Staphylococcus epidermidis & 1 & 0 \\
\hline Streptococcus anginosus & 0 & 1 \\
\hline Streptococcus pneumoniae & 13 & 48 \\
\hline Streptococcus pyogenes & 2 & 3 \\
\hline \multicolumn{3}{|l|}{ (B) Atypical } \\
\hline Achromobacter species & 1 & 0 \\
\hline Acinetobacter species & 1 & 3 \\
\hline Chlamydia pneumoniae & 0 & 3 \\
\hline Citrobacter freundii & 0 & 1 \\
\hline Enterobacteriaceae & 4 & 5 \\
\hline Escherischia coli & 4 & 1 \\
\hline Klebsiella oxytoca & 0 & 2 \\
\hline Klebsiella pneumonia & 2 & 1 \\
\hline Morganella morganii & 1 & 0 \\
\hline Mycobacterium tuberculosis complex & 0 & 1 \\
\hline Mycoplasma pneumoniae & 1 & 2 \\
\hline Proteus mirabilis & 1 & 0 \\
\hline Pseudomonas aeruginosa & 2 & 1 \\
\hline Serratia marescens & 1 & 1 \\
\hline \multicolumn{3}{|l|}{ (C) Other (viral) } \\
\hline Adenovirus & 1 & 1 \\
\hline Cytomegalovirus & 2 & 6 \\
\hline Enterovirus & 0 & 2 \\
\hline Influenza & 0 & 4 \\
\hline Parainfluenza & 0 & 2 \\
\hline Respiratory syncytial virus & 0 & 3 \\
\hline Rhinovirus & 1 & 3 \\
\hline
\end{tabular}

Figure 1 Alphabetical list of all detected microorganisms, quantified and with heat map for both the DS group and the control group of children without underlying disorders.

abnormalities, etc. These are depicted in Table 1 and are similar in both groups, except that more DS children suffer from recurrent lower respiratory tract infections and none of them were suspected to have aspirated a foreign body. As reported previously (8), there is a major difference in prevalence of airway anomalies (in $68.1 \%$ of DS children versus $29.6 \%$ of controls, $\mathrm{P}<0.001)$.

In most patients, cultures showed multiple organisms (for example in $64 \%$ of the DS group). The encountered microorganisms were listed and labeled as typical bacteria, atypical bacteria or other (mostly viruses) (Figure 1), based on experience and previous studies (12-14). We then quantified the numbers of patients in whom all of the individual micro-organisms were found (see Figures 1,2). We attributed each patient to a category, depending on the microbiological data (Figure 1 and Table 2): patients with (I) sterile cultures or only presence of commensals, (II) only typical bacteria present, (III) only atypical microorganisms present, and (IV) a combination of both typical and atypical microorganisms detected. When comparing the DS children to the group of children without underlying conditions, we found similar microorganisms. Also, the percentages of encountered categories were comparable, no statistically significant differences were found (Table 2). We also compared the most frequently reported bacteria with $>10$ patients (i.e., Haemophilus influenzae, Moraxella catarrbalis, Streptococcus pneumoniae and Staphylococcus aureus) by Chi square test. This only showed a higher prevalence of Haemophilus influenzae in the DS group $(\mathrm{P}=0.017)$ but no other significant differences.

\section{Discussion}

Even though children with DS are more prone to infections (often originating in the respiratory tract) than children not affected by DS due to several predisposing factors $(4,5,10)$, this study concludes that the microorganisms encountered in BAL fluid samples are similar. Most frequently, cultures showed growth of typical bacteria such as Haemophilus influenzae, Moraxella catarrbalis and Streptococcus pneumoniae in both groups. This was also the case in several studies of children with protracted bacterial bronchitis (13-15). The previously mentioned case reports about atypical pathogens in DS (16-18) therefore seem to be rather exceptional. When treating patients with DS and (chronic) lower airway infections, antibiotic therapy should not necessarily be adjusted to a broader spectrum than in children without underlying conditions. However, this study did not look at drug resistance patterns, which could be an interesting study subject given the fact that these children receive more (often) antibiotics for various respiratory tract infections (5). Other limitations are that, although we have managed to gather relatively large study populations with deep (bronchoalveolar) sampling, the numbers of specific bacteria and viruses are often too small to compare. The used culture-based techniques are also less sensitive than for example 16S rRNA sequencing for this type of research. Therefore, these conclusions must be approached with caution. Also, the multiple comorbidities in these patients and at times atypical or more severe 


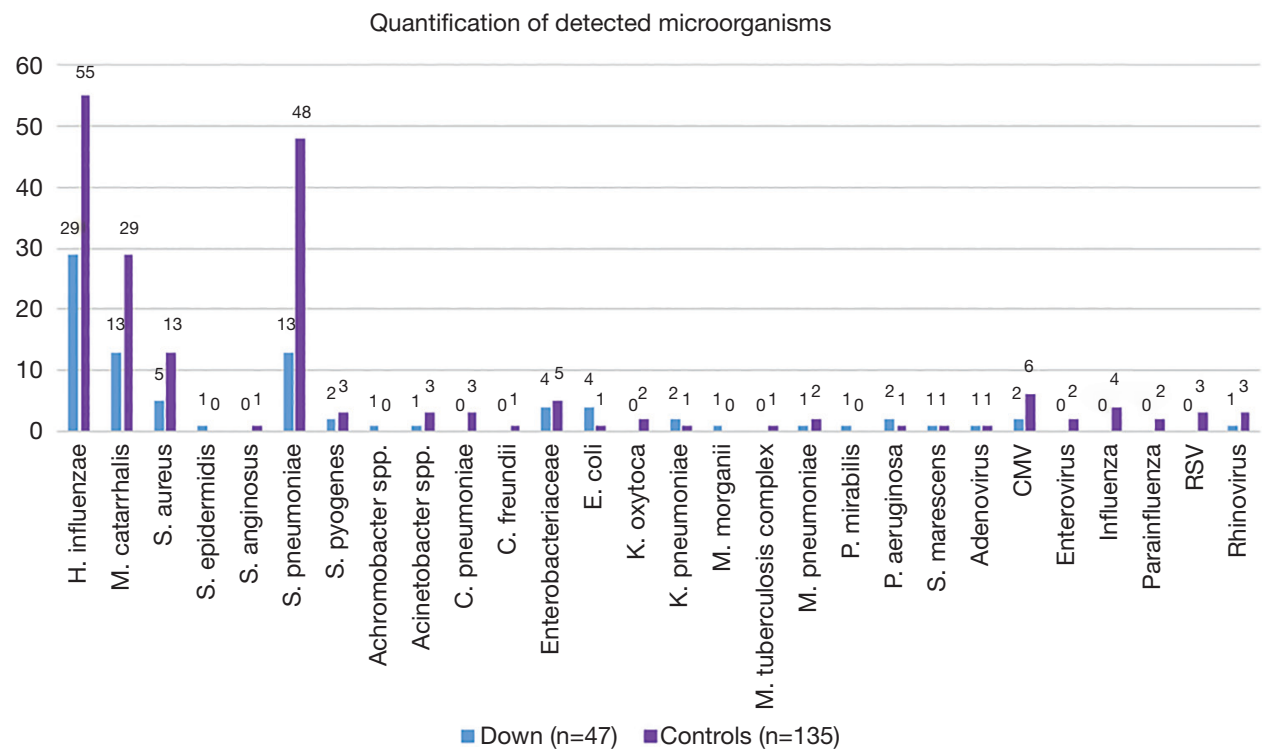

Figure 2 Quantification of detected microorganisms.

Table 2 Comparison of microbiological findings in DS population versus children without underlying conditions

\begin{tabular}{|c|c|c|c|c|}
\hline & Total $(n=182)$ & Down $(n=47)$ & Controls $(n=135)$ & Comparison subgroups ( $\mathrm{P}$ value) \\
\hline \multicolumn{5}{|c|}{ A) Comparison of categories of microorganisms, $n(\%)$} \\
\hline Sterile/commensals & $43(23.6)$ & $7(14.9)$ & $36(26.7)$ & 0.289 \\
\hline Typical bacteria & $86(47.3)$ & $24(51.1)$ & $62(45.9)$ & \\
\hline Atypical bacteria and/or viruses & $18(9.9)$ & 7 (14.9) & $11(8.1)$ & \\
\hline \multicolumn{5}{|c|}{ B) Comparison per microorganism, n (\%) } \\
\hline H. influenza & & & & 0.017 \\
\hline Present & $84(46.2)$ & $29(61.7)$ & $55(40.7)$ & \\
\hline Absent & $98(53.8)$ & $18(38.3)$ & $80(59.3)$ & \\
\hline S. pneumoniae & & & & 0.373 \\
\hline Present & $61(33.5)$ & $13(27.7)$ & $48(35.6)$ & \\
\hline Absent & $121(66.5)$ & 34 (72.3) & $87(64.4)$ & \\
\hline S. aureus & & & & 0.842 \\
\hline Present & 18 (9.9) & 5 (10.6) & $13(9.6)$ & \\
\hline Absent & $164(90.1)$ & $42(89.4)$ & $122(90.4)$ & \\
\hline Others & & & & 0.484 \\
\hline Present & $68(37.4)$ & $20(42.6)$ & $48(35.6)$ & \\
\hline
\end{tabular}

$P$ values calculated by Chi square test, significance level $P \leq 0.05$. 
course of infections vindicate a thorough evaluation and adequate treatment.

\section{Conclusions}

No significant differences were found concerning the lower airway microbiota of children with DS compared to children with similar symptomatology but without underlying conditions. Based on these (albeit limited) data, we conclude that the higher infectious burden in children with DS is not caused by a different microbial composition, but could perhaps be better explained by the immunological and anatomical abnormalities, these topics too deserve more clarification. The type of microorganisms however appears similar.

\section{Acknowledgments}

I would like to thank Dr. S. Van Goethem for helping with the technical information and sharing his passion about microbiology.

Funding: None.

\section{Footnote}

Reporting Checklist: The authors have completed the MDAR reporting checklist. Available at https://dx.doi. org/10.21037/tp-20-460

Data Sharing Statement: Available at https://dx.doi. org/10.21037/tp-20-460

Peer Review File: Available at https://dx.doi.org/10.21037/ tp-20-460

Conflicts of Interest: All authors have completed the ICMJE uniform disclosure form (available at https://dx.doi. org/10.21037/tp-20-460). The authors have no conflicts of interest to declare.

Ethical Statement: The authors are accountable for all aspects of the work in ensuring that questions related to the accuracy or integrity of any part of the work are appropriately investigated and resolved. This study is approved by the Ethics Committee of our institution (Antwerp University Hospital, Belgium; approval number $19 / 17 / 229$ ) and was conducted in accordance with the Declaration of Helsinki (as revised in 2013). Given the data are collected retrospectively and anonymized, informed consent was omitted.

Open Access Statement: This is an Open Access article distributed in accordance with the Creative Commons Attribution-NonCommercial-NoDerivs 4.0 International License (CC BY-NC-ND 4.0), which permits the noncommercial replication and distribution of the article with the strict proviso that no changes or edits are made and the original work is properly cited (including links to both the formal publication through the relevant DOI and the license). See: https://creativecommons.org/licenses/by-nc-nd/4.0/.

\section{References}

1. Stoll C, Dott B, Alembik Y, et al. Associated congenital anomalies among cases with Down syndrome. Eur J Med Genet 2015;58:674-80.

2. Watts R, Vyas H. An overview of respiratory problems in children with Down's syndrome. Arch Dis Child 2013;98:812-7.

3. Bloemers BL, Broers CJ, Bont L, et al. Increased risk of respiratory tract infections in children with Down syndrome: the consequence of an altered immune system. Microbes Infect 2010;12:799-808.

4. Ram G, Chinen J. Infections and immunodeficiency in Down syndrome. Clin Exp Immunol 2011;164:9-16.

5. Alsubie HS, Rosen D. The evaluation and management of respiratory disease in children with Down syndrome (DS). Paediatr Respir Rev 2018;26:49-54.

6. Fitzgerald P, Leonard H, Pikora TJ, et al. Hospital admissions in children with down syndrome: experience of a population-based cohort followed from birth. PLoS One 2013;8:e70401

7. So SA, Urbano RC, Hodapp RM. Hospitalizations of infants and young children with Down syndrome: evidence from inpatient person-records from a statewide administrative database. J Intellect Disabil Res 2007;51:1030-8.

8. De Lausnay M, Verhulst S, Boel L, et al. The prevalence of lower airway anomalies in children with Down syndrome compared to controls. Pediatr Pulmonol 2020;55:1259-63.

9. Bertrand P, Navarro H, Caussade S, et al. Airway anomalies in children with Down syndrome: endoscopic findings. Pediatr Pulmonol 2003;36:137-41.

10. Hamilton J, Yaneza MM, Clement WA, et al. The prevalence of airway problems in children with Down's syndrome. Int J Pediatr Otorhinolaryngol 2016;81:1-4. 
11. Pravit J. Bronchoscopic findings in Down syndrome children with respiratory problems. J Med Assoc Thai 2014;97:S159-63.

12. Wu BG, Segal LN. Lung Microbiota and Its Impact on the Mucosal Immune Phenotype. Microbiol Spectr 2017. doi:10.1128/microbiolspec.BAD-0005-2016.

13. Marsh RL, Smith-Vaughan HC, Chen ACH, et al. Multiple Respiratory Microbiota Profiles Are Associated With Lower Airway Inflammation in Children With Protracted Bacterial Bronchitis. Chest 2019;155:778-86.

14. Ahmed B, Cox MJ, Cuthbertson L, et al. Comparison of the upper and lower airway microbiota in children with chronic lung diseases. PLoS One 2018;13:e0201156.

15. Verhulst S, Boel L, Van Hoorenbeeck K. Protracted

Cite this article as: De Lausnay M, Verhulst S, Boel L, Van Hoorenbeeck K. Lower airway microbiota in children with Down syndrome compared to controls with similar respiratory symptomatology. Transl Pediatr 2021;10(7):1818-1824. doi: $10.21037 /$ tp-20-460 bacterial bronchitis: bronchial aspirate versus

bronchoalveolar lavage findings: a single-centre retrospective study. BMJ Paediatr Open 2019;3:e000507.

16. Cant AJ, Gibson PJ, West RJ. Bacterial tracheitis in Down's syndrome. Arch Dis Child 1987;62:962-3.

17. Orlicek SL, Walker MS, Kuhls TL. Severe mycoplasma pneumonia in young children with Down syndrome. Clin Pediatr (Phila) 1992;31:409-12.

18. Winters JL, O'Connor WN, Broughton RA, et al. Bordetella bronchiseptica pneumonia in a patient with Down syndrome: a case report and review. Pediatrics 1992;89:1262-5. 